\title{
The Indications and Outcomes of Ileostomy Following Pancreas Transplantation
}

\author{
Okechukwu Onuma Okidi*, Maria Mitu Pretorian, Bence Forgacs, Babatunde Campbell and Afshin Tavakoli \\ Transplant Unit, Manchester Royal Infirmary, Central Manchester NHS Foundation Trust, Manchester, United Kingdom
}

*Corresponding author: Okechukwu Onuma Okidi, Transplant Unit, Manchester Royal Infirmary, Central Manchester NHS Foundation Trust, Manchester, United Kingdom, Tel: 07466717556; E-mail: okechukwu.okidi@mft.nhs.uk

Received: 20 Nov, 2018 | Accepted: 21 Jan, 2019 | Published: 28 Jan, 2019

Citation: Okidi OO, Pretorian MM, Forgacs B, Campbell B, Tavakoli A (2019) The Indications and Outcomes of Ileostomy Following Pancreas Transplantation. Transplant Res J 3(1): dx.doi.org/10.16966/2473-1730.119

Copyright: : (C) 2019 Okidi OO, et.al. This is an open-access article distributed under the terms of the Creative Commons Attribution License, which permits unrestricted use, distribution, and reproduction in any medium, provided the original author and source are credited.

\begin{abstract}
Background: Pancreas transplantation is associated with high incidence of intra-abdominal sepsis and reoperations. There is little data on the incidence and need for an ileostomy post pancreas transplantation. However, it is noted anecdotally that this is not uncommon occurrence post enteric drained pancreas transplantation.

Aim: The aim of our study was to investigate the incidence and indications for an ileostomy post pancreas transplantation.

Method: We maintain a prospective data base of patient outcomes and complications following pancreas transplantation in our unit. We looked at the incidence, indications and outcomes of ileostomy post pancreas transplantation.

Result: Between 2001 and January 2010 we performed 210 pancreas transplants. The patient survival at 1 year and pancreas graft survival at 1 year was $92 \%$ and $80 \%$ respectively. Of these, 69 pancreases were bladder drained and 135 were enteric drained. 15 patients required an ileostomy. The indications for an ileostomy were bleeding with intra-abdominal sepsis (6), enteric leak with peritonitis (3), vascular thrombosis (2), severe pancreatitis (3), and severe rejection (1). All patients who had an ileostomy except one had an enteric drained pancreas. The single case of bladder drain that required ileostomy was consequent upon conversion to enteric drain.

Of these 15 patients, 6 died within 1 year and did not have their ileostomy reversed. The death occurred in the early post-op period and was due to sepsis/multiple organ failure (5) and unknown cause (1). The remaining patients all had their ileostomy reversed. The mean length of time between formation and closure of ileostomy was 13 months. There were no reported complications following closure of ileostomy.

Conclusion: An ileostomy is a significant consequence of pancreas transplantation and was required in $7 \%$ of patients in a large series. It is associated with a high morbidity and mortality rate. Although enteric drainage of a pancreas transplant is metabolically preferable, the need for an ileostomy was $11 \%$ in this group. Once patients have recovered from immediate post-operative risk of sepsis, the majority of these ileostomies can be reversed safely with minimal complications. The high incidence of a stoma makes it a prerequisite to inform patients of this possibility pretransplant.
\end{abstract}

\section{Introduction}

Pancreas transplantation is currently an established treatment modality for the insulin dependent diabetic patient. About 1800 pancreas transplants are performed annually worldwide, with average pațient and graft survival rates of $95 \%$ and $85 \%$ respectively [1]. Pancreas transplantation also is now considered a lifesaving procedure [2] and not just a procedure for improving quality of life. Simultaneous pancreas and kidney (SPK) transplantation is currently the gold standard treatment for the Type 1 diabetic with end stage renal failure (ESRF) without any prohibitive cardio-vascular risks. It can improve longevity and quality of life for diabetic patients [3].

The majority of transplant centers presently use enteric drainage (ED) for the diversion of the pancreatic juice thus avoiding complications associated with bladder drainage namely bicarbonate loss, frequent urinary tract infection (UTI), hematuric stone formation, urine leaks and dehydration. However, enteric diversion bears an increased risk of early intra-abdominal infection [4].

Generally, pancreas transplantation is associated with a high rate of post-operative intra-abdominal complications probably because of the peculiar nature of the gland. Interventions to deal with these complications especially in the early post-operative periods often necessitate creating an emergency diverting stoma- ileostomy-(with or without graft pancreatectomy) in order to save patients life.

Ileostomy, particularly in the immunosuppressed such as the post pancreas transplant patient who also often have possible secondary end organ damage from background diabetic mellitus, is associated with a 
higher degree of morbidity and high mortality [5]. The additional cost of prolonged hospital stay and the associated psychological torture are enormous on the patient.

Ileostomy in the post pancreas transplant patient therefore deserves a serious attention. Surprisingly, little or no attention has been given to ileostomy and its predisposing early post-operative surgical complications in pancreas transplantation till date.

We therefore decided in this review to investigate the incidence, indications and outcome of ileostomy in our local pancreas transplantation series.

\section{Methods}

We maintain a prospective data base of patient outcome and complications following pancreas transplantation in our unit. We reviewed these data retrospectively to determine the incidence of ileostomy post pancreas transplantation. We matched the demographics of the donor and recipients, cold ischemic time, type of pancreas transplantation and drainage type between the general pancreas transplant group and those that required ileostomy.

All the pancreas transplant recipients were treated with quadruple immunosuppression which consisted of monoclonal induction therapy, Tacrolimus or Cyclosporin, Mycophenolate or Aziathioprine, and Prednisolone. They all received peri-operative anticoagulation according to existing protocol. Prophylactic Antibiotics and Antifungals were usually given. Total Parenteral Nutrition (TPN) was used in initial post-operative period.

Indications for ileostomy were determined from the early post-operative complications, which necessitated emergency relaparotomies and consequently ileostomies. These indications were categorized accordingly.

Outcome was evaluated in terms of morbidity and mortality.

The indices of morbidity in this study were:

1. Associated graft loss (pancreas $+/$ - kidney)

2. Hospital stay duration

3. Number of re-operations

4. Duration of ileostomy before its takedown

5. Any permanent ileostomy.
The mortality was restricted to deaths within 1 year. Exact time of death and causes were determined.

\section{Results}

Between 2001 and January 2010 we performed 210 pancreas transplants which comprised of 161 simultaneous pancreas and kidney (SPK), 37 pancreas after kidney (PAK) and 12 pancreas transplants alone (PTA)s. Exocrine drainage was: bladder drained (BD)-69; enteric drained (ED)-135, unrecorded drainage type-6. The patient survival at 1 year and pancreas survival at 1 year was $92 \%$ and $80 \%$ respectively. 1-year mortality was $8 \%$ and average duration of hospital stay was 21 days.

15 patients required ileostomy i.e. an incidence rate of $7 \%$ (13 SPK, 1 PAK, 1 PTA). The mean age of these patients was 44 years with a range of 17-53 years. The sex incidence was 8 males and 7 females. The donor-recipient demographics in both ileostomy patients and the general pancreas transplant cohort are comparable as shown in table 1.

The C.I.T. is marginally higher in the patients who required ileostomy but not statistically significant. Majority of the patients who required ileostomy had enteric drainage (ED) i.e. more than $10 \%$ incidence of ileostomy in ED as against $1.4 \%$ of $\mathrm{BD}$. Note that the single case of ileostomy from $\mathrm{BD}$ was consequent upon conversion to $\mathrm{ED}$ in presence of severe pancreatitis.

This is illustrated in figure 1 .

\section{Types of pancreas drainage}

The indications for the re-laparotomies resulting in formation of ileostomy were categorized into the following:

- Bleeding with intra-abdominal sepsis in 6 patients (40\%)

- $\quad$ Enteric leak with peritonitis in 3 patients (20\%)

- Pancreas vascular thrombosis in 2 patients (13\%)

- $\quad$ Severe pancreatitis in 3 patients (20\%)

- $\quad$ Severe rejection in 1 patient $(7 \%)$

The indications are symbolically represented in a pie chart.

Ileostomy was loop ileostomy in all patients except one who had end ileostomy; no reason given for that.

Table 1: Donor Recipient Demographics between lleostomy Patients and General Pancreas Transplant Populations.

\begin{tabular}{|l|c|c|}
\hline & General Pancreas TX population $\mathrm{n}=210$ & $\begin{array}{c}\text { Ileostomy population } \\
\mathrm{n}=15(7 \% \text { of total) }\end{array}$ \\
\hline Recipients: Age- years- mean (range) & $41(15-63)$ & $43.6(17-53)$ \\
Sex -Male no. (\%) & $128(61 \%)$ & $8(53 \%)$ \\
Female no. (\%) & $82(39 \%)$ & $7(47 \%)$ \\
\hline Donors: Age - mean (range) & $31(7-60)$ & $27(10-54)$ \\
BMI*- mean (range) & $23(13.3-30.9)$ & $23.2(17.2-28.3)$ \\
HBD* & $191(91 \%)$ & $15(100 \%)$ \\
NHBD* & $19(9 \%)$ & $0(0 \%)$ \\
\hline Cold Ischemic Time (CIT): minutes-mean (range) & $840(441-1327)$ & $881(680-1014)$ \\
\hline Types of Transplant: SPK* & $161(77 \%)$ & 13 i.e. $8 \%$ of SPK \\
PAK* & $37(17 \%)$ & 1 i.e. 3\% of PAK \\
PTA* & $12(6 \%)$ & 1 i.e. $8 \%$ of PTA \\
\hline Type of Drainage: ENTERIC & $135(64 \%)$ & 14 (>10\% of ED) \\
BLADDER & $69(33 \%)$ & $1(1.4 \%$ of BD) \\
Not Recorded & $6(3 \%)$ & 0 \\
\hline
\end{tabular}

*SPK-Simultaneous Pancreas and Kidney transplant. PAK-Pancreas after kidney transplant. PTA-Pancreas Transplant Alone. BMI-Body Mass Index. HBDHeart Beating Donor. NHBD-Non-Heart Beating Donor. 


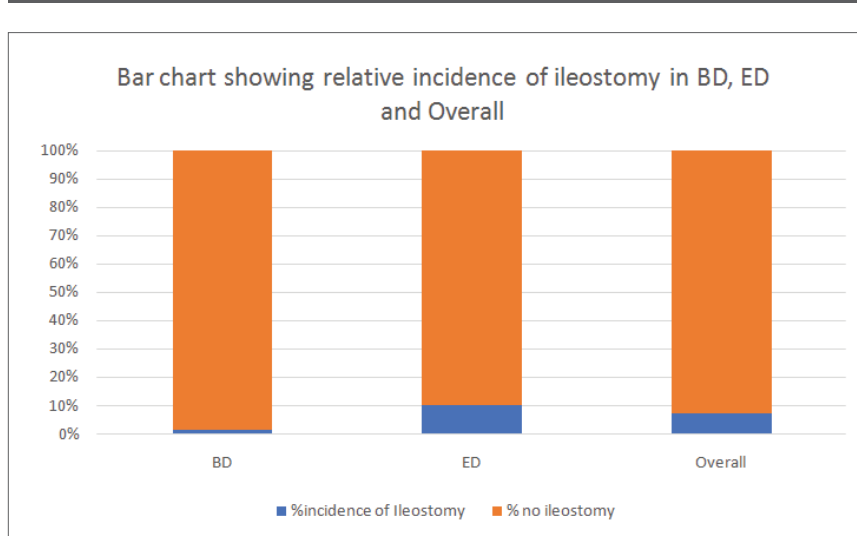

Figure 1: Bar chart showing relative percentage incidence of ileostomy in BD, ED, and Overall.

Outcome was evaluated in terms of morbidity and mortality.

A detailed account is presented in table 2

\section{Footnote}

Re-operations includes following procedures:

- Pancreatectomy + /- nephrectomy

- Ileostomy closure

\section{- Debridement and washout of wounds}

- Exploration and drainage of intra-abdominal collection (abscess/ hematoma)

The average time interval from pancreas transplant to ileostomy was 5 weeks (range $<1-10$ weeks). Of the 15 patients who required ileostomy 11 lost their pancreas grafts as well. One patient lost both pancreas and kidney grafts. Hospital stay was grossly prolonged following ileostomy; average of 88 days (range 28-142 days) and requiring many re-operations to deal with their minor complications.

Average number of reoperations was three (range 1-7). Of the 15 patients, 6 died within 1 year and did not have their ileostomies reversed. The remaining 9 patients all had their ileostomies reversed. The mean length of time between formation and closure of ileostomy was 13 months (range 6-26 months). One patient had his ileostomy closed after 26 months because he was on Sirolimus and had poor wound healing. There were no reported complications following closure of ileostomy.

The one-year peri-operative mortality was 6 out of 15 i.e. $40 \%$. Most of the deaths occurred at about 3 months post-transplant and mostly related to sepsis and multiple organ failure (5) and (1) was of unknown cause. The 1-year patient and pancreas graft survival in patients requiring ileostomy were $60 \%$ and $27 \%$ respectively.

\section{Discussion}

The primary goal of ileostomy in post pancreas transplant patient developing intra-abdominal complications is as a salvage procedure to save the life of patient. Preservation of pancreas graft is of secondary consideration. This is corroborated by Troppman et al. [5] in their submission that once a pancreas graft related complication requiring relaparotomy occurs the focus must shift from graft salvage to preservation of life.

In our practice at the time most of our pancreases were positioned head down with the duodenum anastomosed to either distal ileum in
ED or to bladder in BD. Consequently, on relaparotomies, depending on degree of intra-abdominal sepsis and systemic condition, decision was made to either preserve or remove the pancreas. Graft pancreatectomies were usually always followed with an ileostomy while in graft conservation; ileostomy may be indicated to rest the pancreas as the case may be. In the one case of BD pancreas presenting with hematuria, decision was made to convert to enteric drainage and same time rest the pancreas with a proximal diverting enterostomy i.e. ileostomy because of associated severe pancreatitis.

Majority of the relaparotomies and ileostomies occurred 5-6 weeks post-transplant. Indication of vascular thrombosis presented in less than one week. First was a case of pancreas graft thrombosis presenting 5 days post-transplant and of course ended as graft pancreatectomy with an ileostomy. Second case was due to splenic artery thrombosis, presenting $4^{\text {th }}$ post-operative day and had a successful embolectomy to salvage the pancreas graft. The patient survived after 84 days hospitalization and had the ileostomy reversed 11 months later.

Bleeding with sepsis was actually the most common indication for relaparotomies in our study (40\%); presenting between 3 -and 10 -weeks post-transplant. About half of the cases were bleeding from Mycotic aneurysm in Common Iliac artery (CIA). Ileostomy in these cases was consequent upon graft pancreatectomy. This is not unusual as some other investigators have also reported bleeding from mycotic aneurysm [5]. Three to Ten weeks post- transplant is not too early for Mycotic aneurysm to develop. Donor perfusion fluid may be the source of bacterial contamination at the time of implantation thereby predisposing to development of Mycotic aneurysm in these patients. However, by time of this study we had not adopted our current policy of routinely culturing donor perfusion fluid at time of transplantation, and therefore do not have any data on that. Although none of our patients presented thus but It is pertinent to also note that bleeding from an aneurysm can present as gastro intestinal (GI) hemorrhage from an arterio-enteric fistula in ED pancreases. An example is a case report by Lopez, et al from Lexington Kentucky [6].

Hasse, et al. [7] reported thrombosis and infections as the most frequent causes of technical failure in pancreas transplantation. Ozaki, et al. [8], Eckoff, et al. [9] and Duozdijian, et al. [10] all reported infection, thrombosis and anastomotic leak as the most frequent causes.

Anastomotic leak after pancreas transplant usually occurs early in the post-operative course, but may also be seen late. Late leak is defined as leak occurring more than 3 months post-transplant [11]. Early leaks are usually due to technical complications such as poor blood supply and ischemia of duodenal wall, poor wound healing secondary to aggressive immunosuppressive therapy, trauma, post reperfusion edema or presence of infection around pancreas graft [12]. Possible causes of late leaks include blunt trauma, CMV infection or acute rejection [11]. Our patients with anastomotic leak presented early, at about 2 weeks post-transplant; 2 out of 3 patients just had proximal ileostomy to rest the graft and they did well without need for graft pancreatectomy. The $3^{\text {rd }}$ patient however had the pancreas removed because of associated severe pancreatitis. They all survived and all had their ileostomy reversed 9 -16 months afterwards.

We should mention at this juncture that different authors have described various ways of managing anastomotic/ duodenal leaks post pancreas transplant. Graft pancreatectomy provides definitive, and perhaps the safest treatment, but renders patient insulin dependent. Graft preservation can be considered in presence of limited abdominal contamination or absence of severe pancreatitis. Some pancreas graft 
Table 2: Outcome

\begin{tabular}{|c|c|c|c|c|c|c|c|c|c|}
\hline $\begin{array}{c}\text { Indication } \\
\mathrm{N} \%\end{array}$ & $\begin{array}{l}\text { Time to } \\
\text { Ileostomy }\end{array}$ & Intra Op Findings & $\begin{array}{c}+/- \\
\text { Pancreatectomy }\end{array}$ & $\begin{array}{c}\text { Hospital } \\
\text { Stay (days) }\end{array}$ & $\begin{array}{l}\text { Time to } \\
\text { takedown of } \\
\text { lleostomy }\end{array}$ & $\begin{array}{l}\text { No. of re- } \\
\text { operation }\end{array}$ & $\begin{array}{l}\text { Permanent } \\
\text { Ileostomy }\end{array}$ & $\begin{array}{l}\text { Death } \\
\text { Time/ } \\
\text { cause }\end{array}$ & Comments \\
\hline \multicolumn{10}{|c|}{ 1. BLEEDING with sepsis $6=(40 \%)$. } \\
\hline $\mathrm{i}$ & 10 weeks & Bleeding/ sepsis & + & 122 & 26 months & 5 & - & No & \\
\hline ii. & 5 weeks & Bleeding/ sepsis & + & 98 & - & 5 & - & $\begin{array}{c}3 \text { months } \\
\text { post tx }\end{array}$ & $\begin{array}{c}\text { Reactive } \\
\text { haemorrhage } \\
\text { 2nd to post op } \\
\text { peritonitis }\end{array}$ \\
\hline iii. & 3 weeks & $\begin{array}{l}\text { Bleeding mycotic } \\
\text { aneurysm (R) } \mathrm{CI}^{*}\end{array}$ & + & 105 & - & 2 & - & 3 months & MOD \\
\hline iv. & 6 weeks & $\begin{array}{l}\text { Bleeding mycotic } \\
\text { aneurysm (R) CIA* }\end{array}$ & + & 56 & - & 4 & - & 2 months & $\begin{array}{c}\text { Sudden death? } \\
\text { Cause }\end{array}$ \\
\hline v. *PAK & 6 weeks & $\begin{array}{c}\text { Pancreas abscess/ } \\
\text { bleeding }\end{array}$ & + & 50 & 6 months & 2 & - & No & \\
\hline vi.*PTA & 3 weeks & Mycotic Aneurysm & + End Ileostomy & 30 & 6 months & 1 & - & No & \\
\hline \multicolumn{9}{|c|}{ 2. ENTERIC LEAK with peritonitis $3=(20 \%)$} & \\
\hline i. & $?$ & Anastomotic leak & - & 28 & 16 months & 3 & - & No & \\
\hline ii. & 2 weeks & Anastomotic leak & - & 79 & 9 months & 1 & - & No & \\
\hline iii. & 2 weeks & $\begin{array}{c}\text { Anastomotic leak/ } \\
\text { pancreatitis }\end{array}$ & + & 142 & 14 months & 2 & - & No & \\
\hline \multicolumn{10}{|c|}{ 3. VASCULAR THROMBOSIS $2=(13 \%)$} \\
\hline i. & 5 days & $\begin{array}{l}\text { Pancreas } \\
\text { thrombosis }\end{array}$ & + & 84 & - & 5 & - & 3 months & MOD \\
\hline ii. & ? 4 days & $\begin{array}{l}\text { Splenic artery } \\
\text { thrombosis } \\
\text { embolectomy }\end{array}$ & - & 84 & 11 months & 7 & - & No & $\begin{array}{c}\text { Embolectomy to } \\
\text { salvage graft }\end{array}$ \\
\hline \multicolumn{10}{|c|}{ 4. SEVERE PANCREATITIS $3=(20 \%)$} \\
\hline i. & 3 weeks & Necrosis/ bleeding & + & 90 & 19 months & 2 & - & 3 years & Pneumonia \\
\hline ii. & 10 weeks & Necrosis/bleeding & + & 108 & - & 2 & - & 3 months & $*(\mathrm{ARDS})$ \\
\hline iii. & 4 weeks & $\begin{array}{l}\text { Haematuria/ } \\
\text { pancreatitis }\end{array}$ & - & 115 & 11 months & 3 & - & No & $\begin{array}{c}\text { Conversion from } \\
\text { BD - ED**+ } \\
\text { Ileostomy }\end{array}$ \\
\hline $\begin{array}{l}\text { 5. SEVERE } \\
\text { REJECTION } \\
1=(7 \%)\end{array}$ & 10 weeks & $\begin{array}{l}\text { Severe rejection + } \\
\text { Repeated bleeds/ } \\
\text { sepsis }\end{array}$ & $\stackrel{+}{+}+$ Nephrectomy & 130 & - & 3 & - & 3 months & $\begin{array}{c}\text { MOD } \\
\text { Septicemia }\end{array}$ \\
\hline
\end{tabular}

*PAK-Pancreas after Kidney transplant. PTA-Pancreas Transplant Alone. (R) CIA-Right Common Iliac Artery. MOD-Multiple Organ Dysfunction. ARDSAcute Respiratory Distress Syndrome

**Case of bladder drained pancreas presenting with hematuria and severe pancreatitis; underwent conversion to enteric drainage+diverting proximal stoma (ileostomy) 


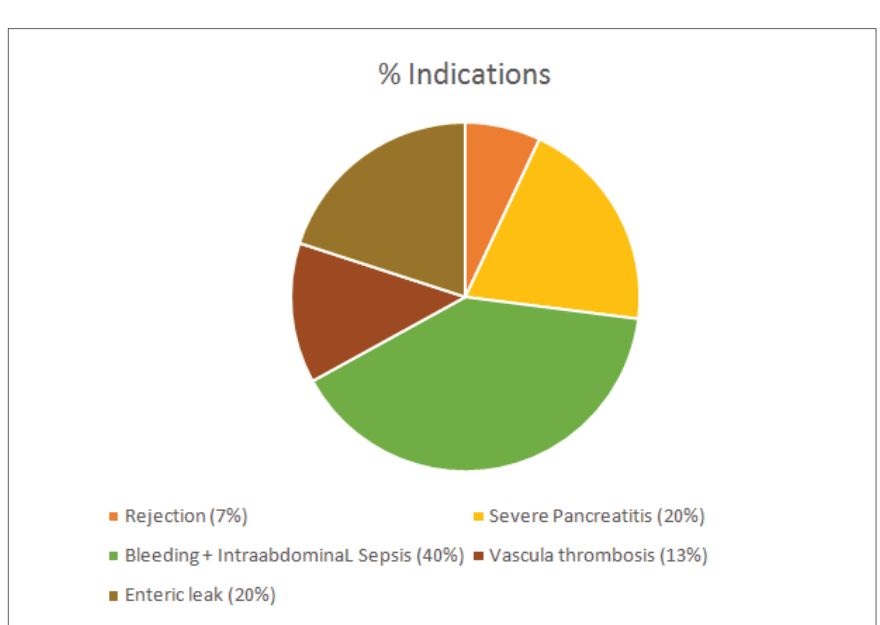

Figure 2: Pie Chart showing indications for ileostomy and their percentage occurrence.

Note: some patients had more than 1 complication at a time.

preservation procedures that have been described for managing anastomotic/duodenal leaks include percutaneous or surgical tube drainage [13], primary surgical repair ${ }^{(13)}$, proximal diversion enterostomy [14], partial or total duodenectomy $+/$ - pancreatic duct occlusion or enteric or cutaneous drainage (wirsugostomy) [15,16]. As emphasized by Boggi, et al. [17] these procedures are generally reserved for stable patients who have limited abdominal contamination. Kumar et al confirms from their experience that tube duodenostomy can be a safe option but can lead to persistent pancreatic fistula. They also advocated for a proximal diversion enterostomy to allow early oral feeding and avoid long term complications associated with parenteral nutrition [14].

We had 3 cases of severe pancreatitis which presented within 3-10 weeks post-transplant; 2 had graft pancreatectomy+ileostomy but unfortunately died, first at about 3 months from ARDS and the second after 3 years from pneumonia. The $3^{\text {rd }}$ case was originally a $\mathrm{BD}$ pancreas which presented with hematuria and severe pancreatitis and was managed by conversion to ED+proximal diverting ileostomy. This patient survived and had the ileostomy reversed after 11 months when inflammatory processes had subsided.

Rejection with repeated bleeds and sepsis was the indication in the last of our patients. This same patient lost both pancreas and kidney grafts and eventually died of multiple organ failure within three months post-transplant.

This raises the question of early diagnosis of rejection in pancreas transplant. Elevation of blood sugar is known to be a late presentation of pancreatic rejection for which salvage of the graft is usually unsuccessful [18]. Ability to diagnose rejection by monitoring urine amylase has been the argument in favor of bladder drainage (BD) of pancreatic juice. However, because of unique morbidity of BD many transplant centers are currently in favor of enteric drainage (ED) [19]. ED is more physiologic. Moreover, several new diagnostic methods of pancreatic rejection have been developed including serum lipase, human anodal trypsinogen, glucose disappearance rate (KG) and serial fine needle aspiration enabling pancreatic rejections regardless of drainage approach [18]. In kidney-Pancreas transplants serum creatinine can be used as surrogate marker of pancreas rejection. More recently several centers have explored the utility of anastomosing the graft duodenum to the recipient duodenum which facilitates endoscopic surveillance biopsies to detect occult rejection, and CMV infection $[20,21]$.

ED though currently favored has been found to be associated with risk of intra-abdominal sepsis [7] and therefore higher rate of ileostomy. In the present study the incidence of ileostomy was approximately $11 \%$ in $\mathrm{ED}$ group as against $1.4 \%$ in $\mathrm{BD}$ group. Essentially all the ileostomies are related to $\mathrm{ED}$ as the single case of $\mathrm{BD}$ required ileostomy only consequent upon enteric conversion.

Other than ED, increased donor age and prolonged cold ischemic time are other factors that have been implicated in relaparotomies post pancreas transplant, but were not reflected in our study. Donor age in both the general pancreas transplant population and those who required ileostomy was comparable. Cold ischemic time though marginally higher in ileostomy group, the difference was not statistically significant. Previous peritoneal dialysis [4] in the pancreas graft recipient is also a known potential cause of intra-abdominal sepsis but it was not investigated in our study.

Pancreas grafts are known to be associated with the highest surgical complication rate of all routinely transplanted solid organs [23]. Ileostomies in post pancreas transplant recipients carry very high morbidity and mortality risks. It has a much higher detrimental effect than seen in other transplant recipients or even nonimmunocompromised patients undergoing major general surgical procedures [5]. Reason for this has been adduced to the background diabetes with possible end-organ damage; exposure to strong immunosuppressive induction agents and the subjection to technically demanding dual organ transplantation [7].

The above reason may explain the poor outcome recorded in our study. Eleven out of the fifteen ileostomy patients had lost their pancreas graft; hospital stay was prolonged to an average of 3 months; had multiple re-operations to deal with other associated complications such as wound infections, abscess collections, fistulas etc. Six out of the fifteen patients died within 1 year i.e. mortality of $40 \%$. One other patient died after 3 years. Majority of the deaths were from sepsis and multiple organ failure, and not so much from stoma fluid imbalance as stomas were essentially not very high-output. The outcome in terms of 1-year patient and 1-year graft survival post ileostomy therefore becomes $60 \%$ and $23 \%$ respectively, which is appalling. Those who survived the critical period of sepsis, all had their ileostomies reversed at an average time interval of 13 months.

\section{Conclusion}

An ileostomy is a significant consequence of pancreas transplantation and was required in $7 \%$ of patients in a large series. Although enteric drainage of a pancreas transplant is metabolically preferable the need for an ileostomy was approximately $11 \%$ in this group. Once patients have recovered from immediate post-operative risks of sepsis, the majority of these ileostomies can be reversed safely with minimal complications.

Efforts should be made to minimize the chances of early surgical complications which often necessitate ileostomies. The high incidence of a stoma makes it a pre-requisite to inform patients of this possibility pre-transplant.

\section{References}

1. International Pancreas Transplant Registry.

2. Gruessner RWG, Serutherland DER (2004) Transplantation of the pancreas. Springer-Verlag New York, Inc. 
3. Waki K, Terasaki PI (2006) Kidney graft and patient survival with and without a simultaneous pancreas utilizing contralateral kidneys from the same donor. Diabetes Care 29: 1670-1672.

4. Steurer W, Tabbi MG, Bonatti H, Spechtenhauser B, Ollinger R, et al. (2002) Stapler duodenojejunostomy reduces intraabdominal infection after combined pancreas kidney transplantation as compared with hand-sawn anastomosis. Transplant Proc 34: 33573360.

5. Troppmann C, Gruessner AC, Dunn DL, Sutherland DE, Gruessner RW (1998) Surgical complications requiring early relaparotomy after pancreas transplantation: a multivariate risk factor and economic impact analysis of the cyclosporine era. Ann Sur 227: 255-268.

6. Lopez NM, Jeon H, Ranjan D, Johnston TD (2004) Atypical etiology of massive gastrointestinal bleeding: arterio-enteric fistula following enteric drained pancreas transplant. Am Surg 70: 529-532.

7. Hesse UJ, Sutherland DE, Simmons RL, Najarian JS (1986) Intraabdominal infections in pancreas transplant recipients. Ann Surg 203: 153-162.

8. Ozaki CF, Stratta RJ, Taylor RJ, Langnas AN, Bynon JS, et al. (1992) Surgical complications in solitary pancreas and combined pancreaskidney transplantations. Am J Surg 164: 546-551.

9. Eckhoff DE, Sollinger HW (1993) Surgical complications after simultaneous pancreas-kidney transplant with bladder drainage. Clin Transpl 185-191.

10. Douzdjian V, Abecassis MM, Cooper JL, Smith JL, Corry RJ (1993) Incidence, management and significance of surgical complications after pancreatic transplantation. Surg Gynecol Obstet 177: 451-456.

11. Nath DS, Gruessner A, Kandaswamy R, Gruessner RW, Sutherland $\mathrm{DE}$, et al. (2005) Late anastomotic leaks in pancreas transplant recipients - clinical characteristics and predisposing factors. Clin Transplant 19: 220-224.

12. Secchi A, Di Carlo V, Martinenghi S, La Rocca E, Caldara R, et al. (1992) Octreotide administration in the treatment of pancreatic fistulae after pancreas transplantation. Transpl Int 5: 201-204.

13. Al-Adra D, McGilvray I, Goldaracena N, Spetzler V, Laurence J, et al. (2017) Preserving the Pancreas Graft: Outcomes of Surgical Repair of Duodenal Leaks in Enterically Drained Pancreas Allografts. Transplant Direct 3: e179.
14. Kumar S, Singh S, Kenwar DB, Rathi M, Bhadada S, et al. (2016) Management of Graft Duodenal Leak in Simultaneous Pancreas Kidney Transplant-a Case Report from India and Review of Literature. Indian J Surg 78: 402-406.

15. Pieroni E, Napoli N, Lombardo C, Marchetti P, Occhipinti M, et al. (2018) Duodenal graft complications requiring duodenectomy after pancreas and pancreas-kidney transplantation. Am J Transplant 18: 1388-1396.

16. Spetzler VN, Goldaracena N, Marquez MA, Singh SK, Norgate A, et al. (2015) Duodenal leaks after pancreas transplantation with enteric drainage - characteristics and risk factors. Transpl Int 28: 720-728.

17. Boggi U, Vistoli F, Del Chiaro M, Moretto C, Croce C, et al. (2010) Total duodenectomy with enteric duct drainage: a rescue operation for duodenal complications occurring after pancreas transplantation. Am J Transplant 10: 692-697.

18. Prieto $M$, Sutherland $D E$, Goetz FC, Rosenberg ME, Najarian JS (1987) Pancreas transplant results according to the technique of duct management: bladder versus enteric drainage. Surgery 102: 680-691.

19. Kuo PC, Johnson LB, Schweitzer EJ, Bartlett ST (1997) Simultaneous pancreas/kidney transplantation--a comparison of enteric and bladder drainage of exocrine pancreatic secretions. Transplantation 63: 238-243.

20. Gunasekaran G, Wee A, Rabets J, Winans C, Krishnamurthi V (2012) Duodenoduodenostomy in pancreas transplantation. Clin Transplant 26: 550-557.

21. Horneland R, Paulsen V, Lindahl JP, Grzyb K, Eide TJ, et al. (2015) Pancreas transplantation with enteroanastomosis to native duodenum poses technical challenges--but offers improved endoscopic access for scheduled biopsies and therapeutic interventions. Am J Transplant 15: 242- 250.

22. Corry RJ, Nghiem DD, Schulak JA, Beutel WD, Gonwa TA (1986) Surgical treatment of diabetic nephropathy with simultaneous pancreatic duodenal and renal transplantation. Surg Gynecol Obstet 162: $547-555$

23. Guessner AC, Sutherland DE (1994) Pancreas transplant results in the United Network for Organ Sharing (UNOS) United States of America (USA) Registry compared with non-USA data in the International Registry. Clin Transpl 47-68. 